\title{
Late Post-traumatic Epilepsy in Children and Young Adults: Impropriety of Long-Term Antiepileptic Prophylaxis and Risks \\ in Tapering
}

Sandra Strazzer Marco Pozzi Paolo Avantaggiato Nicoletta Zanotta Roberta Epifanio Elena Beretta Francesca Formica Federica Locatelli Sara Galbiati Emilio Clementi Claudio Zucca

\author{
Abstract \\ Background After traumatic brain injury, epilepsy affects \\ up to $20 \%$ of children. It is a risk factor, for both clinical \\ recovery and cognitive performance; therefore pharmacological \\ therapy is advisable. Current guidelines recommend \\ prophylaxis to be initiated as soon as possible and tapered \\ 1 week after trauma. However, no guideline exists for \\ paediatric patients and the clinical practice is \\ heterogeneous. \\ Objective In our institute, prophylaxis was routinely \\ tapered 6 months after trauma. Therefore we investigated \\ whether this prophylaxis or its tapering influenced the \\ development of post-traumatic epilepsy, together with \\ several clinical-demographic factors. \\ Methods The study population comprised all patients \\ with post-traumatic brain injury referred to this institute \\ between 2002 and 2009 who consented to participate. \\ Clinical, epileptological and pharmacological data were \\ collected. The role of prophylaxis and several other predictors \\ on occurrence of post-traumatic epilepsy was \\ analysed through logistic regressions. \\ Results Two hundred and three patients (145 paediatric) \\ were followed for 57 months on average. Risk factors for \\ epilepsy were past neurosurgery [odds ratio $(\mathrm{OR})=2.61$, \\ $95 \%$ confidence interval (CI) 1.15-5.96], presence of \\ epileptiform anomalies (OR $=6.92,95 \%$ CI 3.02-15.86) \\ and the presence of prophylaxis (OR $=2.49,95 \% \mathrm{CI}$ \\ 1.12-5.52), while higher intelligence quotient (IQ) was \\ protective $(\mathrm{OR}=0.96,95 \% \mathrm{CI} 0.95-0.98)$. While evaluating \\ possible different effects within and after 6 months \\ (tapering, for those under prophylaxis), we found that \\ epileptiform anomalies (OR = 7.61, $95 \%$ CI 2.33-24.93, \\ and $\mathrm{OR}=8.21,95 \% \mathrm{CI} 3.00-22.44)$ and IQ (OR = 0.96, \\ $95 \%$ CI 0.94-0.98, and OR = 0.97, $95 \%$ CI 0.95-0.98) \\ were always significant predictors of epilepsy, while neurosurgery \\ (OR $=4.38,95 \%$ CI 1.10-17.45) was significant \\ only within 6 months from trauma, and prophylaxis \\ (OR $=3.98,95 \%$ CI 1.62-9.75) only afterwards. \\ Conclusions These results suggest that prophylaxis was \\ irrelevant when present; furthermore its tapering increased \\ the risk of epilepsy. Since the presence of epileptiform \\ anomalies was the main predictor of post-traumatic epilepsy, \\ such anomalies may be useful to better direct the \\ choice of prophylaxis.
}

Drug prophylaxis against late post-traumatic epilepsy, maintained for 6 months after trauma, was not efficacious.

Tapering prophylaxis 6 months after trauma 
increased the occurrence of late post-traumatic epilepsy. We hypothesise that longer prophylaxis may be useful.

Among the risk factors identified for late posttraumatic epilepsy, the presence of electroencephalography (EEG) epileptiform anomalies is the strongest.

The choice of prescribing, maintaining and tapering drug prophylaxis against late post-traumatic epilepsy may be taken based on the presence of EEG epileptiform anomalies.

\section{Introduction}

Traumatic brain injury (TBI) is a serious public health concern. It is associated with a high mortality rate and long-term neurological sequelae including motor, cognitive, behavioural and language deficits [1]. Among these complications is post-traumatic epilepsy (PTE), representing up to $20 \%$ of all cases of symptomatic epilepsy and $5 \%$ of all cases of epilepsy. The incidence of PTE worldwide ranges between 9 and $42 \%$ amongst civilians, and around $50 \%$ in cases of penetrating brain injury $[2,3]$. PTE is also a frequent complication in children with severe TBI, ranging between 10 and $20 \%$, and is often drug resistant $[4,5]$. A common set of definitions for posttraumatic epileptic seizures adopted by many researchers is the following: immediate seizures, which occur less than $24 \mathrm{~h}$ after injury; early post-traumatic seizures (EPTS), which occur less than 1 week after injury; and late posttraumatic seizures (LPTS), which occur more than a week after injury [6, 7]. The diagnosis of PTE is usually formulated for patients who exhibit two or more unprovoked LPTS [8]. While there is consensus on the fact that the EPTS and PTE need to be treated pharmacologically, reports about antiepileptic prophylaxis in the literature do not provide a conclusive approach regarding drugs of choice and treatment duration. The current guidelines concur in defining phenytoin-based antiepileptic prophylaxis to be efficacious in the early post-traumatic phase, i.e. from trauma (as soon as possible) up to 1 week after trauma; antiepileptic prophylaxis should be tapered thereafter [9-12]. Considering the medium-long term, there is inconsistent evidence to support either a beneficial or a detrimental effect of antiepileptic prophylaxis [9-18]. The above indications were drawn from studies conducted on adult patients with severe brain trauma and diverse followup durations. These conclusions cannot be directly transferred to the paediatric practice, especially in a rehabilitation setting, where the potential damage caused by seizures in patients recovering from TBI is sizeable [19]. In rehabilitation, it may be reasonable to maintain antiepileptic prophylaxis as a precaution for periods longer than 1 week, in the absence of contraindications, pharmacological interactions or adverse effects [20-22]. While safety parameters are clearly defined, the efficacy of antiepileptic prophylaxis has been defined as seizure-suppressing activity during therapy, or this plus a persistent anti- epileptogenic effect maintained even after drug tapering and discontinuation $[23,24]$. Both effects are 
often impractical to demonstrate, because of the requirement of long follow-up periods and because of possible confounding effects of other concomitant or subsequent therapies, treatments and medically relevant events. In addition, it should be considered that even patients who experienced no EPTS may still be prescribed antiepileptic prophylaxis, even long term.

As we mostly receive patients from intensive care units later than 1 week after TBI, in our rehabilitation unit, we routinely tapered antiepileptic prophylaxis at 6 months after trauma. To evaluate whether this alternative approach could be useful for paediatric/young patients with TBI, we investigated the influence of antiepileptic prophylaxis and non-pharmacological factors on the occurrence of PTE.

\section{Materials and Methods}

\subsection{Setting and Patients}

The Acquired Brain Injury Unit of the Scientific Institute IRCCS E. Medea provides rehabilitation to children and young adult patients (usually of 30 years or less), whether the injury is post-traumatic or due to other brain lesions. For this retrospective cohort study, inclusion criteria were as follows: being hospitalised in the unit between 2002 and 2009; authorising data collection for this study by informed consent. This period was chosen since antiepileptic prophylaxis tapering was performed strictly at 6 months after TBI only until 2009. Exclusion criteria were as follows: age at TBI of more than 30 years, presence of non-traumatic brain lesions, presence of past neurological disorders, follow-up duration less than 12 months, and antiepileptic prophylaxis tapering at a time different than 6 months after TBI. Patients were followed as long as they S. Strazzer et al. attended periodic rehabilitation controls, up to July 2014. This study was notified to the local Ethics Committee.

\subsection{Data Collection}

The occurrence of PTE was recorded alongside its time of occurrence. PTE was the study endpoint as long as it happened before July 2014. Patients who incurred no PTE until July 2014 achieved study completion without reaching the endpoint. The following data were also collected for each patient: age at TBI, sex, severity of trauma according to the Glasgow Coma Scale (GCS), type of TBI (closed or open head injury, when the cranial fracture was documented by X-ray or computed tomography (CT) scan), post-traumatic neurosurgery, type of brain lesion as established by magnetic resonance imaging (MRI) or CT scan, duration of unresponsiveness, electroencephalography (EEG) identification of epileptiform anomalies, presence of antiepileptic prophylaxis and drugs used. The disability level was evaluated by Glasgow Outcome Score (GOS) at the first discharge from rehabilitation, and the intelligence quotient (IQ) score was assessed during the first month of rehabilitation by age-appropriate scales. For statistical purposes, when the cognitive neurological picture was severe, patients were given an IQ of 19, indicating a profound cognitive impairment (following diagnostic and statistical manual of mental disorders, 4th edition (DSM-IV)).

2.3 Antiepileptic Drug Prophylaxis 
All antiepileptic prophylaxes were prescribed and started in intensive care units within $24 \mathrm{~h}$ from trauma. Therapies were chosen based only on clinical judgment. Clinicians of intensive care units chose which drug to use, its dose calculated in $\mathrm{mg} / \mathrm{kg}$ (following labelling indications), and whether to maintain antiepileptic prophylaxis at discharge from intensive care. Our rehabilitation unit received patients with pre-set prophylactic therapies and did not change them; we only monitored plasma drug concentrations in order to adjust therapies in case of under/over-dosing. Since patients were mostly admitted to rehabilitation with an antiepileptic prophylaxis on-going for more than 1 week (as decided in intensive care in contrast with standing guidelines [9-12]), prophylaxis in our unit was routinely maintained stable for 6 months before tapering. Tapering of antiepileptic prophylaxis was agreed upon by the parents and/or the patients, it was performed gradually over a period of 4 months while monitoring the EEG status, and it was reverted in case of seizure onset. Patients who were not on prophylaxis were monitored by periodic EEGs and antiepileptic therapy was started only after two seizures. For study comparisons, treatment should be interpreted as follows: during the time frame within 6 months from TBI, one group of patients was under prophylaxis and one group without it, allowing evaluation of the effect of antiepileptic prophylaxis; during the time frame after 6 months from TBI, the group on prophylaxis tapered it, allowing evaluation of the effect of tapering from antiepileptic prophylaxis.

2.4 Statistical Analyses Statistical analyses were conducted using SPSS version 22 (IBM, Chicago, IL, USA). Categorical variables were described as numbers with percentages and compared by Pearson's v2 tests. Continuous variables were described as means with standard deviation (SD) and compared by T tests. $\mathrm{p}$ values of $\backslash 0.05$ (two-tailed) were considered significant, and post hoc comparisons between groups were Bonferronicorrected. Multivariate logistic regressions were initially carried out with a full set of predictors by "enter" approach. The following dependent variables were analysed: occurrence of PTE (no/yes-binary logistic regression); occurrence of PTE in time (no PTE as reference/PTE within 6 months/PTE after 6 months-multinomial logistic regression); use of antiepileptic prophylaxis (no/yes-binary logistic regression). Independent variables entered in the regression models were IQ, GOS, GCS, duration of coma, age at TBI, site of the lesion (not identified/focal/lobar/multilobar hemispheric/multifocal), presence of epileptiform anomalies (no/yes), presence of EPTS (no/yes), type of trauma (closed/open), past neurosurgery (no/yes), male sex (no/yes); plus, for PTE prediction, presence of antiepileptic prophylaxis (no/yes). For every significant predictor, odds ratios (ORs) were calculated, together with $95 \%$ confidence intervals (CIs) and corresponding $\mathrm{p}$ values. For the sake of clarity, results were shown from "stepwise forward" regression models ( $p$-in $\backslash 0.05$, p-out $[0.1$, using the above order of predictors), which selected only significant predictors, maintaining ORs similar to the initial full models.

\section{Results}


Within the study time frame 203 patients with TBI met the inclusion criteria, and 145 (71.4\%) of them were paediatric. Overall, patients were followed for an average of 57 months from TBI (SD 45 months). Demographic data of the study population are shown in Table 1, comparing control patients without PTE $(140,69.0 \%)$ to patients who had PTE within 6 months from TBI $(24,11.8 \%)$ or after 6 months from TBI $(39,19.2 \%)$. During follow-up, seven patients $(3.4 \%)$ displayed single LPTS that did not progress to PTE, and were therefore treated as controls.

Patients were predominantly males $(143,70.4 \%)$ and their age at trauma ranged from 0.48 to 30 years, being lower among patients with PTE occurring in the first 6 months. Based on GCS scores, 187 patients (92\%) suffered severe TBI (\8), 12 (6\%) moderate (9-12), and four (2\%) mild (13-15), with no difference among groups. The mean GOS at the first discharge from the unit was 3.6 (moderatesevere disability), and all patients with PTE had lower scores. Eighty-three patients (40.9\%) had an IQ of [70, 77 (37.9\%) had one between 70 and 20 , and 43 (21.2\%) were assigned an IQ of 19 because of extreme cognitive impairment; patients who incurred PTE had lower overall scores. Most patients displayed multifocal brain injury (96, $47.3 \%) ; 34$ (16.8\%) had hemispheric injury and 27 $(13.3 \%)$ lobar; five patients had no clear damage site. Eighty-two patients (40.4\%) had an open-head trauma; neurosurgery was performed on 107 patients (52.7\%) overall, and significantly more among those who incurred PTE. In total, 11 patients (5.4\%) displayed EPTS, and at the first EEG performed in the unit, 56 patients $(27.6 \%)$ displayed interictal epileptic anomalies, predominantly those who had PTE. Seventy-six patients $(37.4 \%)$ received prophylaxis, and its use resulted to be predominant among patients who suffered PTE after 6 months from TBI, but not among those with earlier PTE. Fifty-three patients (69.7\%) took phenobarbital for antiepileptic prophylaxis, seven (9.2\%) phenytoin, and five (6.6\%) carbamazepine, while six (7.9\%) assumed other monotherapies and five (6.6\%) assumed polytherapies.

Possible risk factors for PTE were first analysed regardless of onset time (binary logistic regression with Pearson's v2 goodness-of-fit $\mathrm{p}=0.26$, Cox and Snell's pseudo-R2 $=0.36$ ), finding significant risk factors in the presence of past neurosurgery $(\mathrm{OR}=2.61,95 \% \mathrm{CI}$ $1.15-5.96, p=0.022)$, the presence of epileptiform anomalies (OR $=6.92,95 \%$ CI 3.02-15.86, $\mathrm{p} \backslash 0.001$ ) and the presence of antiepileptic prophylaxis $(\mathrm{OR}=2.49$, $95 \%$ CI 1.12-5.52, $\mathrm{p}=0.025)$, while higher IQ was protective $(\mathrm{OR}=0.96,95 \%$ CI 0.95-0.98, $\mathrm{p} \backslash 0.001)$. We next tested whether the occurrence of PTE within or after 6 months from TBI (coincident with tapering, in patients who assumed prophylaxis) could be influenced differently (multinomial logistic regression with Pearson's V2 goodness-of-fit $\mathrm{p}=0.12$, Cox and Snell's pseudo$\mathrm{R}_{2}=0.40$ ). The risk of PTE occurring within 6 months from TBI was increased by the presence of past neurosurgery $(\mathrm{OR}=4.38,95 \%$ CI 1.10-17.45, $\mathrm{p}=0.036)$ and the presence of epileptiform anomalies (OR $=7.61,95 \%$ CI 2.33-24.93, $\mathrm{p}=0.001$ ), while higher IQ was protective 
(OR $=0.96,95 \%$ CI $0.94-0.98, \mathrm{p} \backslash 0.001)$; of note, the presence of prophylaxis had no significant effect within 6 months from TBI (OR $=1.03,95 \%$ CI 0.33-3.21, $p=0.961$ ). The risk of PTE occurring after 6 months from TBI was increased by the presence of epileptiform anomalies (OR $=8.21,95 \% \mathrm{CI} 3.00-22.44, \mathrm{p} \backslash 0.001$ ) and by the presence of antiepileptic prophylaxis (OR $=3.98,95 \%$ CI 1.62-9.75, $\mathrm{p}=0.003)$, while higher IQ was protective $(\mathrm{OR}=0.97,95 \% \mathrm{CI} 0.95-0.98$, $\mathrm{p} \backslash 0.001)$. We finally checked whether the effect of antiepileptic prophylaxis on the occurrence of PTE could be redundant, i.e. mediated by clinical factors already comprised in the above models, by evaluating the predictors of prophylaxis (binary logistic regression with Pearson's v2 goodness-of-fit $p=0.49$, Cox and Snell's pseudo$\left.R_{2}=0.17\right)$. We found that the use of antiepileptic prophylaxis was predicted by the occurrence of EPTS

$(\mathrm{OR}=21.71,95 \%$ CI 2.39-197.34, $\mathrm{p}=0.006)$, by the presence of an open head trauma (OR $=2.24,95 \% \mathrm{CI}$ $1.18-4.28, p=0.014$ ), and by the presence of multilobar hemispheric damage (OR $=3.14,95 \%$ CI 1.09-9.02, $\mathrm{p}=0.034$ ); therefore none of the predictors of PTE were also predictors of antiepileptic prophylaxis. A summary of significant predictors is reported in Table 2.

4 Discussion

The long-term efficacy of prophylactic treatments for PTE is not yet clear $[10,12,14,17]$ and no study has clearly established the effects of tapering antiepileptic prophylaxis $[22,23]$, so its optimal duration is approximate [19-21]. Several clinical variables are crucial for PTE development, acting as confounders and reducing the consistency of results from different clinical studies [17, 24]. Furthermore, the involvement of different medical teams during the various stages of the disease affects the standardisation of clinical decisions; for this reason, antiepileptic prophylaxis for PTE is still widely prescribed and maintained long term. A long-term prophylactic treatment, although not supported by current guidelines, may be appropriate if its adverse effects are minimal, which may be the case with conventional anti-epileptic drugs (AEDs) and accurate patient monitoring [12,14].

In this study on paediatric and young adult patients we evaluated the effect of prolonged antiepileptic prophylaxis for PTE and of its tapering at 6 months after injury, controlling for confounders with a control group who received no antiepileptic prophylaxis. Because of the predominance of paediatric patients, the main drug used for antiepileptic prophylaxis was phenobarbital, rather than phenytoin, in consideration of its indications of use and of its easier clinical management and favourable safety profile [25-27]. We confirmed the influence of several factors previously identified by different studies, both considering the overall occurrence of PTE and the occurrence with respect to the 6-month time point, which corresponded to the time of tapering of antiepileptic prophylaxis in treated patients. Interictal epileptiform anomalies resulted to be a reliable and weighty predictor of PTE in every analysis we Risk of Tapering of Long-Term Antiepileptic Prophylaxis for Paediatric Post-traumatic Epilepsy conducted. Puzzlingly, EEG is widely used in clinical 
practice, but epileptiform anomalies are not yet recognised as biomarkers for the risk of PTE after TBI [3, 7, 28, 29]. In view of the literature and of our results, the clinical routine may benefit from considering epileptiform anomalies as a core risk factor for PTE. This would allow a better identification of patients at risk of PTE and guide the choice of prescribing and maintaining antiepileptic prophylaxis. On the contrary, the presence of EPTS did not predict PTE, thereby questioning the role of early seizures as a biomarker for late seizures. Patients who underwent posttraumatic neurosurgery (either one or more interventions) were exposed to additional risks of PTE, a finding partially consistent with the literature $[28,30]$; in fact, we demonstrated an effect on the overall prevalence of PTE, but time-wise analyses evidenced an effect of neurosurgery only within the first 6 months after trauma. Almost onethird of our paediatric patients with a documented cranial fracture did not undergo neurosurgery. The cranial bone of children and young people is thinner and more prone to fractures than that of adults, even with weaker impact forces [31]; therefore the need for neurosurgery may not be strongly associated with severe damage in children as it is in adults, reducing the reliability of neurosurgery as a predictor of PTE in this sample. A protective role was found for higher IQ scores. This finding supports the widespread concept that cognitive impairment is comorbid with PTE [32], due to either seizures or drug toxicity. Moreover, the finding that IQ levels may predict PTE risk could be interpreted in an inverse manner: patients with greater cognitive/neurological disability are at increased risk of epilepsy [8]. As our results may partially depend on the population we studied, it is worth mentioning additional clinical factors that resulted to be associated with the development of PTE, although not being significant predictors (see Table 1). Patients with PTE had lower GOS and longer coma duration, both referring to a deeper impairment of the neural networks. The lower the GOS, and the longer the unresponsiveness period, the higher the patients' neurological severity, including the occurrence of PTE. Lower age was also a factor associated with PTE within the first 6 months after trauma, and interestingly $50 \%$ of children under 3 years developed epilepsy (data not shown). This observation supports the notion that trauma to the immature brain contributes to a greater impairment, by mechanisms such as mossy fibre sprouting during recovery (hallmark of the chronic epileptic brain) that may lead to an imbalance between excitatory and inhibitory synapses, an event at the basis of seizures [29, 33]. These findings indicate that PTE in our sample occurred in younger and more severe patients.

While correcting for the above confounders, we confirmed also in our study population that the antiepileptic prophylaxis they received is not protective against PTE when at maintenance doses, and we documented for the first time that its tapering at 6 months from trauma increases the risk of PTE. These results are consistent with previous reports from the literature [34-36] and provide further warning against the unconditional use of long-term prophylaxis for PTE. Still, the lack of a treated group who 
did not taper antiepileptic prophylaxis leaves an uninvestigated issue; it is unknown whether the extension of antiepileptic prophylaxis for more than 6 months is connected with the same issues we documented, or whether at later time points antiepileptic prophylaxis could acquire a protective role or its tapering could be safe. Theoretically, the results we observed might also depend on the fact that the risk of PTE may still be high at 6-12 months after trauma, a bias that we attempted to control by using a long follow-up period, 4-5 years on average. Nevertheless, what we observed is that an antiepileptic prophylaxis lasting 6 months was twice detrimental, as it did not significantly protect against PTE while present; moreover its tapering increased the risk of PTE. This is further proof that antiepileptic prophylaxis acts by the suppression of ongoing seizures and does not prevent the epileptogenic mechanisms that occur after TBI $[22,23]$. Our sample was epileptologically representative of the general population with PTE. This is a strength of this study; the proportion of EPTS is consistent with data from the literature [2], and we found $31 \%$ of patients with PTE, which is in line with several reports $[2,8,28]$. Furthermore, when we assessed which clinical variables could predict the use of antiepileptic prophylaxis (EPTS, open-head trauma and the presence of hemispheric lobar lesions), we found no superimposition with factors that predicted PTE. Thus we excluded also this type of confounding factors and confirmed that the effects of antiepileptic prophylaxis on PTE were truly drug dependent.

The findings we report may be limited by several characteristics of our study sample. Patients suffered severe TBI and neurological impairment, with long-lasting unresponsiveness periods; the prevalence of neurosurgical interventions was very high, and the neuro-radiological picture severe. Moreover, additional confounders may have been missed. It must be noted that only a randomised controlled study may conclusively ascertain causal relationships between the use of antiepileptic prophylaxis and the occurrence of PTE that our study suggests. Another limitation is that the efficacy of antiepileptic prophylaxis clearly depends upon drugs used. Phenobarbital was commonly used for our patients, since in Europe it is approved for paediatric use with no age restriction. In addition, we receive patients from intensive care units with pre-set therapies, and usually we do not switch drugs. These two reasons prevented the study of newer, potentially better, approaches, such as off-label paediatric uses of levetiracetam or lacosamide.

\section{Conclusion}

This study demonstrates that antiepileptic prophylaxis after TBI, using phenobarbital and other drugs for 6 months, is not efficacious against PTE in children and adults of 30 years or less. However, we observed an increase in the risk of PTE after the prophylaxis was tapered 6 months after TBI, suggesting that it may be best continued in patients who received it. Indeed, antiepileptic prophylaxis is still a choice frequently taken in intensive care units for paediatric patients with TBI, who may have high risks of PTE also after 2-3 months or more, and is frequently 
maintained for several months or years. What could be done, from a clinical perspective? Surely, the standing guidelines should be followed regarding short-term antiepileptic prophylaxis, which is protective and has a positive risk-benefit ratio. Accordingly, antiepileptic prophylaxis should not be maintained in patients who develop no PTE in the first week and show no epileptiform abnormalities. While our work could not investigate shortterm antiepileptic prophylaxis, it provides interesting results regarding patients who are discharged to rehabilitation with an antiepileptic prophylaxis already on-going for more than 1 week. There are two possibilities: either to taper the antiepileptic prophylaxis, in order to comply to the guidelines as soon as possible; or to maintain the antiepileptic prophylaxis for at least 2 years in the absence of seizures, just like a pharmacological treatment for epilepsy. This crucial choice may be based on EEG data, in particular the presence and degree of interictal epileptiform abnormalities, which we identified as a strong biomarker of PTE.

\section{Compliance with Ethical Standards}

Funding This work was supported by Agenzia Italiana del Farmaco and the Italian Ministry of Health (Ricerca Corrente 2016, to S.S. and E.C. and C.Z.). The funding public institutions had no role in any part of the work.

\section{Conflict of interest}

Sandra Strazzer reports no conflicts of interest,

Marco Pozzi reports no conflicts of interest, Paolo Avantaggiato reports no conflicts of interest, Nicoletta Zanotta reports no conflicts of interest, Roberta Epifanio reports no conflicts of interest, Elena Beretta reports no conflicts of interest, Francesca Formica reports no conflicts of interest, Federica Locatelli reports no conflicts of interest, Sara Galbiati reports no conflicts of interest, Emilio Clementi reports no conflicts of interest, and Claudio Zucca reports no conflicts of interest.

\section{Ethical approval}

All procedures involving human participants

were performed in accordance with the ethical standards of the institutional committee and with the 1964 Helsinki declaration and its later amendments or comparable ethical standards. When clinical data were collected, informed consent to anonymise them and use them for research purposes was obtained from all individual participants included in the study. For this type of retrospective study, formal approval from the institutional committee is not required; only a notification of usage of anonymised data is needed.

\section{References}

1. Dikmen SS, Machamer JE, Powell JM, et al. Outcome 3 to 5 years after moderate severe traumatic brain injury, associated mortality and risk factor. Arch Phys Med Rehabil. 2003;84:1449-57.

2. Agrawal A, Timothy J, Pandit L, et al. Post-traumatic epilepsy: an overview. Clin Neurol Neurosurg. 2006;108:433-9.

3. Yeh CC, Chen TL, Hu CJ, et al. Risk of epilepsy after traumatic brain injury: a retrospective population-based cohort study. J Neurol Neurosurg Psychiatry. 2013;84:441-5. 4. Appleton RE, Demellweek C. Posttraumatic epilepsy in children, requiring inpatient rehabilitation following head injury. J Neurol Neurosurg Psychiatry. 2002;72:669-72.

Risk of Tapering of Long-Term Antiepileptic Prophylaxis for Paediatric Post-traumatic Epilepsy 5. Barlow KM, Spowart JJ, Minns RA. Early posttraumatic seizures in nonaccidental head injury: relation to outcome. Dev Med Child Neurol. 2000;42:591-4.

6. Annegers JF, Grabow RV, Groover RV, et al. Seizure after head trauma: a population study. Neurology. 1980;30:683-9. 
7. Lowenstein DH. Epilepsy after head injury: an overview. Epilepsia. 2009;50:4-9.

8. Frey LC. Epidemiology of posttraumatic epilepsy: a critical review. Epilepsia. 2003;44:11-7.

9. Colorado Division of Workers' Compensation. Traumatic brain injury medical treatment guidelines. Denver (CO): Colorado Division of Workers' Compensation; 2012 Nov 26, p 119. Available at: https://www.guideline.gov/content.aspx?id=43752. 10. Adelson PD, Bratton SL, Carney NA, et al. Guidelines for the acute medical management of severe traumatic brain injury in infants, children, and adolescents. Chapter 19. The role of antiseizure prophylaxis following severe pediatric traumatic brain injury. Pediatr Crit Care Med. 2003;4:S72-5. 11. Chang BS, Lowenstein DH. Quality Standards Subcommittee of the American Academy of Neurology. Practice parameter: antiepileptic drug prophylaxis in severe traumatic brain injury: report of the Quality Standards Subcommittee of the American Academy of Neurology. Neurology. 2003;14:10-6.

12. Bratton SL, Chestnut RM, Ghajar J, et al. Brain Trauma Foundation; American Association of Neurological Surgeons; Congress of Neurological Surgeons; Joint Section on Neurotrauma and Critical Care, AANS/CNS; Guidelines for the management of severe traumatic brain injury. XIII. Antiseizure prophylaxis. J Neurotrauma. 2007;24:S83-6.

13. Schierhout G, Roberts I. Prophylactic antiepileptic agents after head injury: a systematic review. J Neurol Neurosurg Psychiatry. 1998;64:108-12.

14. Iudice A, Murri L. Pharmacological prophylaxis of post-traumatic epilepsy. Drugs. 2000;59:1091-9.

15. Torbic H, Forni AA, Anger KE, et al. Use of antiepileptics for seizure prophylaxis after traumatic brain injury. Am J Health Syst Pharm. 2013;70:759-66.

16. Szaflarski JP, Nazzal Y, Dreer LE. Post-traumatic epilepsy: current and emerging treatment options. Neuropsychiatr Dis Treat. 2014;10:1469-77.

17. Weston J, Greenhalgh J, Marson AG. Antiepileptic drugs as prophylaxis for post-craniotomy seizures. Cochrane Database Syst Rev. 2015;3:CD007286.

18. Thompson K, Pohlmann-Eden B, Campbell LA, et al. Pharmacological treatments for preventing epilepsy following traumatic head injury. Cochrane Database Syst Rev. 2015;8:CD009900.

19. Mazzini L, Cossa FM, Angelino E, et al. Posttraumatic epilepsy: neuroradiologic and neuropsychological assessment of long-term outcome. Epilepsia. 2003;44:569-74.

20. Grisar T, Bottin P, De Borchgrave D'Altena V, et al. Prophylaxis of the epilepsies: should anti-epileptic drugs be used for preventing seizures after acute brain injury? Acta Neurol Belg.

2005;105:5-13.

21. Young B, Rapp RP, Norton JA, et al. Failure of prophylactically administered phenytoin to prevent post-traumatic seizures in children. Childs Brain. 1983;10:185-92.

22. Janz D. Prognosis and prophylaxis of post-traumatic epilepsy. Boll Lega It Epil. 1982;39:37-41.

23. Dichter MA. Posttraumatic epilepsy: the challenge of translating discoveries in the laboratory to pathways to a cure. Epilepsia. 2009;50:41-5.

24. Temkin NR. Preventing and treating posttraumatic seizures: the human experience. Epilepsia. 2009;50:10-3.

25. Beghi E. Overview of studies to prevent posttraumatic epilepsy. Epilepsia. 2003;44:21-6.

26. Zhang LL, Zeng LN, Li YP. Side effects of phenobarbital in

epilepsy: a systematic review. Epileptic Disord. 2011;13:349-65.

27. Brodie MJ, Kwan P. Current position of phenobarbital in epilepsy and its future. Epilepsia. 2012;53:40-6.

28. Ijff DM, Aldenkamp AP. Cognitive side-effects of antiepileptic drugs in children. Handb Clin Neurol. 2013;111:707-18.

29. Englander J, Bushnik T, Duong TT, et al. Analyzing risk factors for late posttraumatic seizures: a prospective, multicenter investigation. Arch Phys Med Rehabil. 2003;84:365-73.

30. Statler KD. Pediatric posttraumatic seizures: epidemiology, 
putative mechanisms of epileptogenesis and promising investigational progress. Dev Neurosci. 2006;28:354-63.

31. Jensen FE. Introduction. Posttraumatic epilepsy: treatable epileptogenesis. Epilepsia. 2009;50:1-3.

32. Muñoz-Sa'nchez MA, Murillo-Cabezas F, Cayuela A, et al. The significance of skull fracture in mild head trauma differs between children and adults. Childs Nerv Syst. 2005;21:128-32.

33. Asikainen I, Kaste M, Sarna S. Early and late posttraumatic seizures in traumatic brain injury rehabilitation patients: brain injury factors causing late seizures influence of seizures on longterm outcome. Epilepsia. 1998;40:584-9.

34. Hunt RF, Boychuk JA, Smith BN. Neural circuit mechanisms of post-traumatic epilepsy. Front Cell Neurosci. 2013;7:89.

35. Formisano R, Barba C, Buzzi MG, et al. The impact of prophylactic treatment on post-traumatic epilepsy after severe traumatic brain injury. Brain Inj. 2007;21:499-504.

36. Verellen RM, Cavazos JE. Post-traumatic epilepsy: an overview. Therapy. 2010;7:527-31. 